\title{
Dual use of electronic cigarettes and cigarettes
}

Sharon Cox

1 London South Bank University

Concurrent use by a person of electronic cigarettes (e-cigarettes) and cigarettes.

Synonyms: Dual use. 